\title{
A Deep Inference System for the Modal Logic S5
}

\author{
Phiniki Stouppa *
}

March 1, 2006

\begin{abstract}
We present a cut-admissible system for the modal logic $S 5$ in a formalism that makes explicit and intensive use of deep inference. Deep inference is induced by the methods applied so far in conceptually pure systems for this logic. The system enjoys systematicity and modularity, two important properties that should be satisfied by modal systems. Furthermore, it enjoys a simple and direct design: the rules are few and the modal rules are in exact correspondence to the modal axioms.
\end{abstract}

Keywords modal logic S5, proof theory, deep inference, calculus of structures, cut-admissibility.

\section{Introduction}

The failure of the sequent calculus to accommodate cut-admissible systems for the important modal logic $S 5$ (e.g. in Ohnishi and Matsumoto [21]) has led to the development of a variety of new systems and calculi. A partial solution to this problem has been presented in Shvarts [25] and Fitting [5], where theorems of $S 5$ are embedded into theorems of cut-free systems for $K 45$. These systems provide proof search procedures for $S 5$, they are, however, systems of a weaker logic.

Complete solutions to the problem have been mainly obtained via two techniques. The first one concerns the annotation of formulae with information related to Kripke-frame semantics. This information is usually given by means of labels or indices (e.g. in Kanger [16], Mints [18], Simpson [26], Negri [20]). The second technique concerns the exhibition of formulae at

\footnotetext{
${ }^{*}$ Institut für Informatik und angewandte Mathematik, University of Bern. This work has been completed while studying at the International Centre for Computational Logic, Technische Universität Dresden.
} 
syntactic positions on which rules in usual Gentzen systems do not operate (e.g. in Sato [24], Indrzejczak [11], Avron [1], Wansing [30]). These positions may be obtained by adding new structural connectives to the usual sequents. Systems built on the latter technique are conceptually pure, meaning that their data structures correspond to modal formulae of $S 5$. For this reason, this technique is in general preferable over the former.

A closer inspection on those systems shows that all of them overcome the lack of cut-admissibility of the system for $S 5$ in the sequent calculus, following the same principle: the modal rules for $S 5$ presented by Ohnishi and Matsumoto [21] ${ }^{1}$

$$
\frac{\alpha, \diamond \Gamma_{1}, \square \Delta_{1} \vdash \diamond \Gamma_{2}, \square \Delta_{2}}{\diamond \alpha, \diamond \Gamma_{1}, \square \Delta_{1} \vdash \diamond \Gamma_{2}, \square \Delta_{2}}(\diamond \vdash) \quad \frac{\diamond \Gamma_{1}, \square \Delta_{1} \vdash \diamond \Gamma_{2}, \square \Delta_{2}, \alpha}{\diamond \Gamma_{1}, \square \Delta_{1} \vdash \diamond \Gamma_{2}, \square \Delta_{2}, \square \alpha}(\vdash \square)
$$

have the condition that all side formulae must be prefixed with a modality. This restriction makes the cut-rule necessary in proofs of theorems like theorem $B: p \supset \square \diamond p$. The new systems allow derivations, the premise and conclusion of which only partially match their corresponding in one of the above rules, as the condition on side formulae is not satisfied. To illustrate this, we present the cut-free proof of axiom $B$ in the hypersequent system for $S 5$ (see Avron [1]) (left) and its proof with cut in the sequent system (right):

$$
\begin{aligned}
\frac{p \vdash p}{p \vdash \diamond p}(\vdash \diamond) & \\
\frac{p \vdash \mid \vdash \diamond p}{p \vdash \mid \vdash \square \diamond p}(\vdash \square) & \frac{p \vdash p}{p \vdash \diamond p}(\vdash \diamond) \frac{\diamond p \vdash \diamond p}{p_{p} \vdash \square \diamond p}(\vdash \square) \\
\frac{p \vdash \square \diamond p \mid \vdash \square \diamond p}{p \vdash \square \diamond p \mid p \vdash \square \diamond p}\left(W_{r}\right) & \frac{p \vdash \square \diamond p}{\vdash p \supset \square \diamond p}(\vdash \supset) \\
\frac{p \vdash \square \diamond p}{\vdash p \supset \square \diamond p}(\vdash \supset) &
\end{aligned}
$$

In the first proof, the partial matching of the $(\vdash \square)$-rule given above is revealed by the derivation obtained when one removes the topmost and lowest rule applications from it (i.e. the rules $(\vdash \diamond)$ and $(\vdash \supset)$ ): each of the sequents $p \vdash \diamond p$ and $p \vdash \square \diamond p$ matches the principal formula in the premise and conclusion of the rule, respectively, the condition on side formulae is, however, not satisfied.

\footnotetext{
${ }^{1}$ We present their equivalent symmetric variants .
} 
The admissibility of the cut rule in those systems relies strongly on such partial matchings. Evidently, in the above example the premise and conclusion of the derivation match one of the premises and the conclusion of the cut-rule (in the proof to the right), respectively. An overview of the systems and details on how they allow such partial matchings can be found in Stouppa [28].

Consequently, the systems provide mechanisms that allow, in certain cases, deeper applications of the specific rules, so that the application affects only a subsequent of a given sequent. Therefore, the formulation of cut-free systems for $S 5$ requires a technique to apply rules deeper on data structures. Systems with rules that are applicable at any depth enjoy a form of deep inference $^{2}$. Thus, such systems allow nested structures of unbounded depth. Among the existing systems for $S 5$, only the system in display logic (Wansing [30]) enjoys deep inference. The rest allow only nested structures of bounded $\mathrm{depth}^{3}$. However, they have rules that are applicable at every syntactic position. Such a rule is for instance the weakening, which in all systems (of bounded depth) has two versions, one for each position.

Although deep inference is not necessary for just a cut-free formulation for $S 5$, its application allows for some proof theoretical advantages: both the system in display logic and the system we are going to present next, enjoy systematicity and modularity with respect to their modal rules. Systematicity refers to a clear technique for formulating the modal rules out of the modal axioms, and modularity to that every axiom corresponds to a finite number of rules. These properties have been introduced in Wansing [30] and are among those that modal systems should satisfy, since they are strongly related to the generality of a calculus. When one is concerned with the latter, deep inference seems to be necessary as the only existing conceptually pure system for $B$ is the one in display logic (Wansing [30]) and enjoys deep inference. On the other hand, rule applications in deep systems can be combined in richer ways than in sequent systems and therefore, new techniques related to proof search procedures are required.

In the following section we present a system for $S 5$ in a deep inference formalism, the calculus of structures. The formalism accommodates, among others, systems for classical logic (Brünnler [4]), as well as systems for different variants of linear logic (Straßburger [29], Guglielmi and Straßburger

\footnotetext{
${ }^{2}$ Usually deep inference is applied on a calculus rather than on a particular system and is used as a synonym to the calculus of structures. In this case though, it serves solely as a property of a system.

${ }^{3}$ The multiple sequent calculus allows for nested structures of unbounded depth; however, for simplification these have been dropped from the system for $S 5$ (Indrzejczak [11]).
} 
[8]) and several normal modal logics (Stewart and Stouppa [27]). The system for $S 5$ presented here, is a conservative extension of the system for $S 4$ presented in Stewart and Stouppa [27], with rules that correspond to axiom 5. Apart from the properties described above, this system enjoys a simple design with few rules, and a direct way of formalizing the modal rules out of

their axioms: for every axiom $R \supset T, \frac{S\{R\}}{S\{T\}}$ is a rule of the system. Also, all rules can be restricted in such a way that their applications affect only a bounded portion of the data structure. This property is called locality (see Brünnler [4]) and induces a bounded computational cost for rule applications. The local system obtained remains simple, although the number of rules is increased.

Cut-admissibility is presented in section 3 and has been obtained via embedding of cut-free proofs from the hypersequent system for $S 5$. The latter, as well as other hypersequent systems for non-classical logics, can also be embedded into systems in the display logic, as the encoding of hypersequents into display sequents in Wansing [31] suggests. Finally, section 4 summarizes our achievements and the possible research directions that can strengthen the current results.

\section{The System}

The calculus of structures is a proof theoretical formalism introduced by Guglielmi [7] that makes explicit and intensive use of deep inference: in this formalism, all inference rules are granted with deep applicability. It is a generalization of the one-sided sequent calculus, with formulae and sequents being indistinguishable. Thus, all the connectives that appear in proofs are logical ones and inference rules are defined only in terms of formulae. We start with the syntactic presentation of modal formulae in the calculus of structures:

Definition 1 Formulae in modal KS systems are built up as follows:

$$
S::=f f|\boldsymbol{t}| a|\bar{a}|[S, S]|(S, S)| \square S \mid \diamond S,
$$

where the units $\mathrm{ff}$ and $\boldsymbol{t}$ stand for falsity and truth, the schematic letters $a, b, \ldots$ and $\bar{a}, \bar{b}, \ldots$ for atoms and their complements, $\left[S_{1}, S_{2}\right]$ and $\left(S_{1}, S_{2}\right)$ for disjunction and conjunction, and $\square S$ and $\diamond S$ for the usual modal operators. The formula context $S\{-\}$ denotes a formula in which a positive occurrence of a subformula is replaced by -, the hole, and the formula $S\{R\}$ is obtained by filling that hole with the formula $R$. Also, formulae of the form 
$\left[S_{1},\left[S_{2}, \ldots,\left[S_{n-1}, S_{n}\right] \ldots\right]\right]$ are denoted by $\left[S_{1}, S_{2}, \ldots, S_{n}\right]$ and those of the form $S\left\{\left[S_{1}, \ldots, S_{n}\right]\right\}$ by $S\left[S_{1}, \ldots, S_{n}\right]$. The analogous conventions are also applied for conjunction.

Inference rules, rule applications and derivations are defined similarly to those in the sequent calculus, with the distinction that now they range over structures rather than sequents:

Definition 2 Modal structures are the classes of formulae obtained modulo the equations of

- associativity: $\quad[R,[T, U]]=[[R, T], U], \quad(R,(T, U))=((R, T), U)$

- commutativity: $[R, T]=[T, R], \quad(R, T)=(T, R)$

- identity: $\quad R=[R, f f], \quad R=(R, \boldsymbol{t}), \quad \boldsymbol{t}=\square \boldsymbol{t}, \quad f f=\diamond f f$

and the replacement theorem: If $R$ and $T$ are equivalent then so are $S\{R\}$ and $S\{T\}$, for any formula context $S\{-\}$.

Usually structures are denoted by one of their constitutive formulae and so, a structure $R$ denotes the class of formulae that are equivalent to formula $R$ (according to the above equations). Similarly, the structure $\bar{R}$ denotes the class of formulae that are equivalent to the complement of $R$ (in negation normal form). Also, contrary to the sequent calculus, inference rules are now deep and have precisely one premise. Thus, an inference rule is of the form $\frac{S\{R\}}{S\{T\}}$, and the tree-like notation on derivations is replaced by a linear one. For instance, a derivation $\Delta$ with the structures $S_{1}$ and $S_{2}$ as premise and conclusion respectively, takes the form $\|_{\Delta}$. Proofs are all derivations with $\mathbf{t t}$ as a premise. As usual, derivations can be combined sequentially: $\Delta_{1} ; \Delta_{2}$ denotes the derivation obtained by extending $\Delta_{1}$ with $\Delta_{2}$, provided that the conclusion of $\Delta_{1}$ and the premise of $\Delta_{2}$ coincide. Moreover, given a formula context $S\{-\}, S\{\Delta\}$ denotes the derivation obtained from $\Delta$ by replacing every structure $R$ in it with the structure $S\{R\}$.

Another characteristic of the calculus is that in every symmetric, non cut-free system the dual rules are also rules of the system. The dual of a rule is obtained by reversing and negating its premise and conclusion. This symmetry is best esteemed in the case of the cut rule $i \uparrow \frac{S(R, \bar{R})}{S\{\mathrm{ff}\}}$, which 


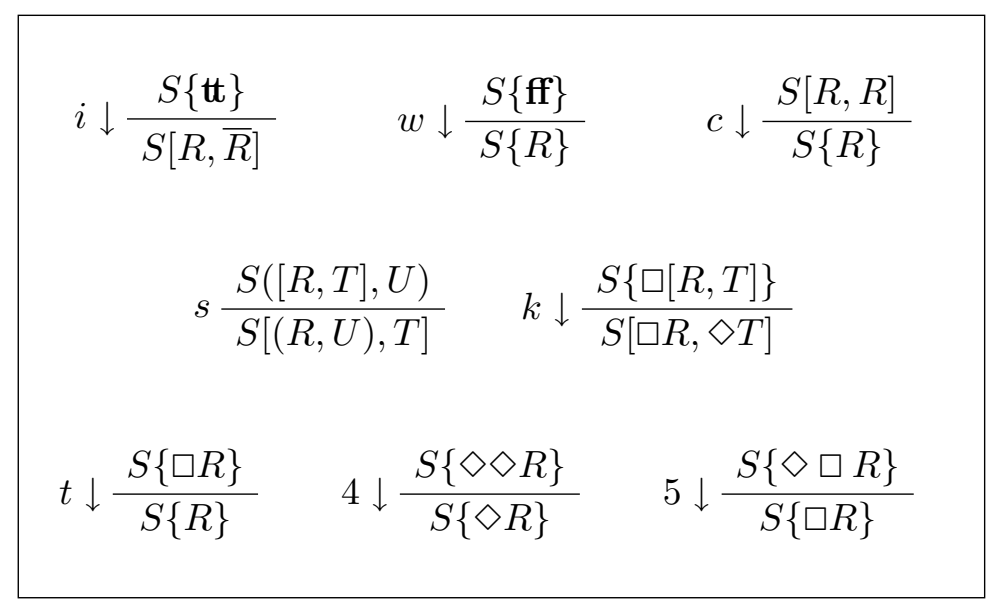

Figure 1: System KSg-S5

is the dual of the interaction rule $i \downarrow$ (Figure 1), the rule that generalizes the sequential axiom-rule.

The rules of the symmetric system for $S 5$, system SKSg-S5, are the rules of system SKSg-S4 (Stewart and Stouppa [27]) and the rules that correspond to axiom 5:

$$
5 \downarrow \frac{S\{\diamond \square R\}}{S\{\square R\}} \quad \text { and } \quad 5 \uparrow \frac{S\{\diamond R\}}{S\{\square \diamond R\}}
$$

Theorem 1 System $\boldsymbol{S K S \boldsymbol { S }} \mathbf{S 5}$ is a system for the logic S5. The cut rule is admissible in this system.

Cut-admissibility is a consequence of the admissibility of all the up rules, the up-fragment, and is obtained by embedding proofs of the hypersequent system for $S 5$, system GS5, in our system. The proof is presented in the next section. Figure 1 shows the cut-free, asymmetric system obtained by removing the up-fragment, system KSg-S5.

Theorems of both systems are their provable formulae, that is, the conclusions of all their proofs. For instance, theorem $B$ can be proved in any of them as follows:

$$
\begin{aligned}
i & \downarrow \frac{\mathrm{t}}{[\diamond \square \bar{a}, \square \diamond a]} \\
5 & \downarrow \frac{[\square \bar{a}, \square \diamond a]}{} \quad t \downarrow \frac{[\bar{a}, \square \diamond a]}{}
\end{aligned}
$$


Equivalence to S5. Equivalence of system SKSg-S5 to the logic S5 is obtained from the equivalence of system $\mathbf{S K S g - S 4}$ to the Hilbert axiomatization of $S 4$ (see Stewart and Stouppa [27]) in a straightforward way. The latter has been obtained in two steps: firstly, by proving that for every rule $\frac{S\{R\}}{S\{T\}}$ of the system, $\vdash S\{R\}_{i} \supset S\{T\}_{i}$ is a theorem of $S 4$ (for all formulae $S\{R\}_{i}$ and $S\{T\}_{i}$ in structures $S\{R\}$ and $S\{T\}$, respectively) ${ }^{4}$. Secondly, by showing that the structures ${ }^{5}$ that correspond to the axioms of $S 4$ are provable in the system and all proofs are closed under modus ponens and necessitation. For instance, in the case of modus ponens, given $\Pi_{1}$ and $\Pi_{2}$ proofs of $[\bar{R}, T]$ and $R$ respectively, we build a proof of $T$ as follows:

$$
\begin{gathered}
\mathbb{t t} \\
s \frac{([\bar{R}, T], R)}{[(R, \bar{R}), T]} \\
T \uparrow \frac{\Pi_{1} ; S\left\{\Pi_{2}\right\} \quad \text { with } S\{-\}=([\bar{R}, T],-) .}{}
\end{gathered}
$$

For the equivalence of SKSg-S5 to $S 5$ we only need to enrich the above results with the proofs of (i) $\vdash \diamond \square R \supset \square R$ and $\vdash \diamond R \supset \square \diamond R$ being theorems of $S 5$ and (ii) the structure that corresponds to axiom 5 being provable in SKSg-S5. The first part follows trivially since the two formulae are the

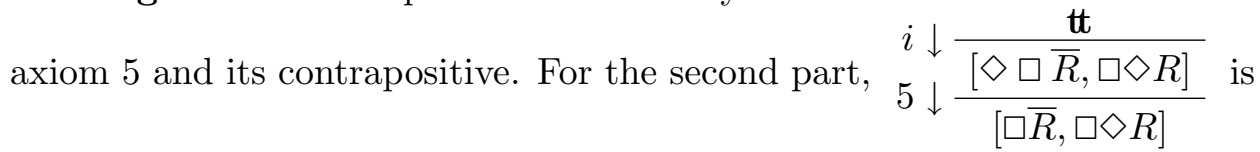
a proof of the structure in consideration.

\section{Cut-admissibility}

As mentioned above, cut-admissibility for SKSg-S5 is the result of the admissibility of its up-fragment. This is obtained via translations of cut-free proofs from the hypersequent system for $S 5$, system GS5, to system $\mathbf{K S g}$ S5 (the cut-free system). The first admits cut-elimination (see Avron [1])

\footnotetext{
${ }^{4}$ An iteration of modus ponens applications on $t$ and the theorems obtained results $\vdash S\{T\}_{i}$, as required.

${ }^{5}$ These structures are obtained via the function ()$^{s}$, which maps a Hilbert formula $\alpha$ to the structure of the formula obtained by replacing every connective in the negation normal form of $\alpha$ with its notational variant in the calculus of structures. For the definition see Stewart and Stouppa [27].
} 
and the translations of its cut-free proofs do not introduce any up-rule applications. As a consequence we obtain a completeness result for the system KSg-S5. We start with a short presentation of system GS5.

Hypersequents and S5. The method of hypersequents is an extension of the sequent calculus, with data structures (called hypersequents) being finite (multi)sets of normal sequents. For instance,

$$
\Gamma_{1} \vdash \Delta_{1}\left|\Gamma_{2} \vdash \Delta_{2}\right| \Gamma_{3} \vdash \Delta_{3}
$$

is a hypersequent of three sequents, seperated by $\mid$. The symbol $\mid$ has a disjunctive reading: a hypersequent is provable if at least one of its sequents is provable.

The system for S5, system GS5 ( Avron [1]), is shown in Figure 2. Formulae are denoted by the schematic letters $\alpha, \beta, \ldots$, (multi)sets of formulae by $\Gamma, \Delta, \ldots$ and (multi)sets of sequents by $G, H, \ldots$. All logical rules and the cut-rule are formulated as in Gentzen system $L K$, with the addition that they are applicable on sets of sequents. For instance, the

rule $\frac{\alpha, \Gamma \vdash \Delta}{\Gamma \vdash \Delta, \neg \alpha}(\vdash \neg)$ takes the form $\frac{G|\alpha, \Gamma \vdash \Delta| H}{G|\Gamma \vdash \Delta, \neg \alpha| H}(\vdash \neg)$. Moreover, every structural rule (i.e. weakening and contraction) admits two versions:

1. the internal version, which is applied on formulae inside a sequent. These are the standard Gentzen rules, which are obtained in the same way logical rules are obtained, as described above.

2. the external version which is applied on sequents. For example, the external version of the contraction $(C$ ext) duplicates a sequent.

The modal rules consist of the modal rules for $S 4$ (rules $(\square \vdash)$ and $(\vdash \square)$ ) and the modalized splitting rule $(M S)$. The notation $\square \Gamma$ is an abbreviation for the set $\{\square \alpha \mid \alpha \in \Gamma\}$. As usual, a proof is a derivation starting with an axiom.

Completeness for GS5 is shown using the translation $\phi_{G}$ of a hypersequent $\mathrm{G}$ to a formula of modal logic, which is defined as follows:

Definition 3 The translation $\phi_{G}$ of a hypersequent $G$ to a modal formula is recursively defined as follows:

- $\phi_{\emptyset}=\perp$ 


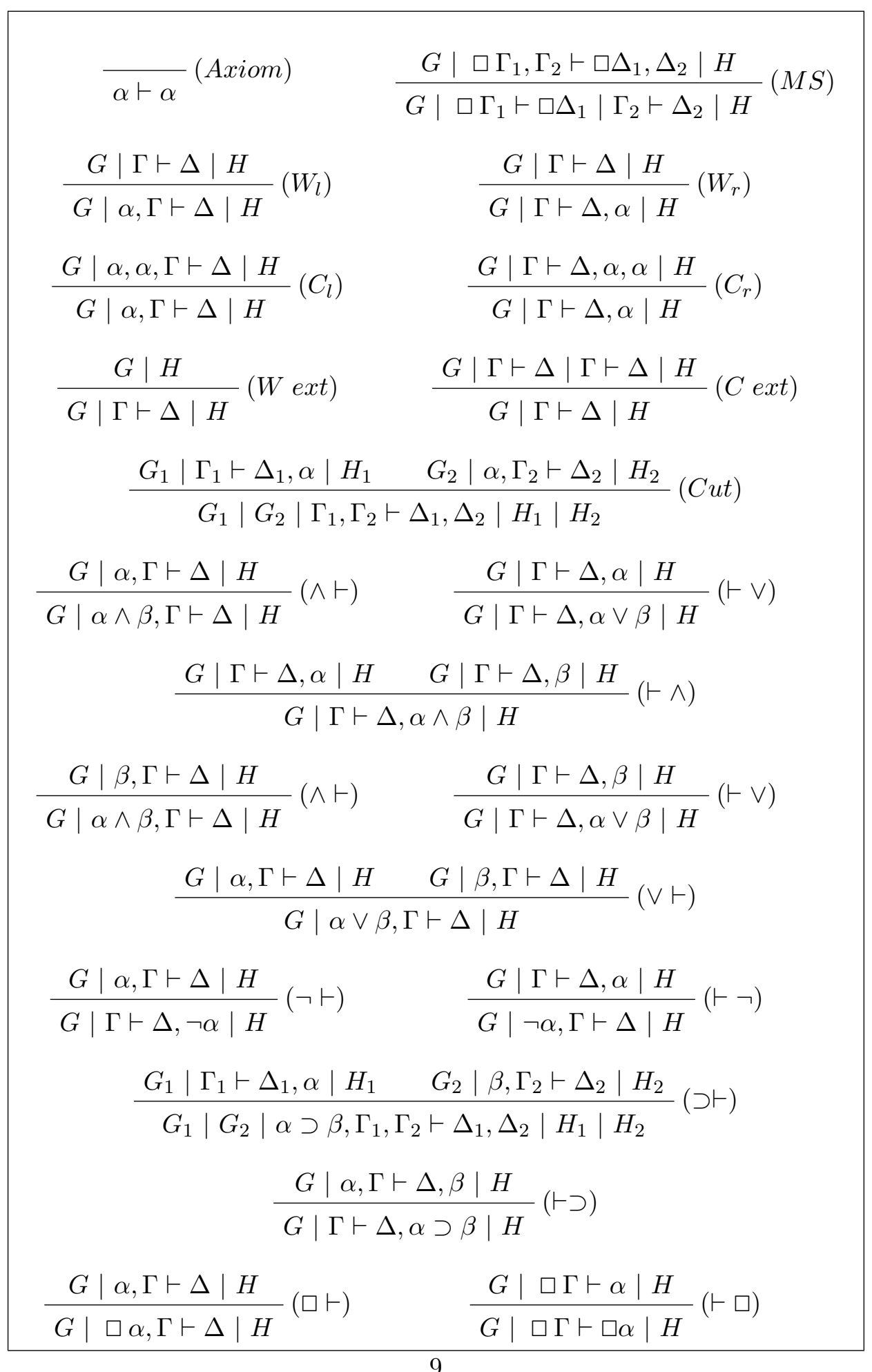

Figure 2: System GS5 
- $\phi_{G_{1}|\Gamma \vdash \Delta| H_{1}}=\phi_{G_{1}} \vee \square \psi_{\Gamma \vdash \Delta} \vee \phi_{H_{1}}$,

where $\psi_{S}$ is the translation of a sequent $S$ to a modal formula:

$$
\begin{aligned}
& -\psi_{\emptyset \vdash \emptyset}=\perp \\
& -\psi_{\alpha, \Gamma \vdash \Delta}=\neg \alpha \vee \psi_{\Gamma \vdash \Delta} \\
& -\psi_{\Gamma \vdash \Delta, \alpha}=\alpha \vee \psi_{\Gamma \vdash \Delta}
\end{aligned}
$$

We can now state the equivalence of GS5 to $S 5$ and the cut-elimination theorem:

Proposition $1 \vdash_{G S 5} G$ iff $\vdash_{S 5} \phi_{G}$.

Theorem 2 System $\boldsymbol{G S 5}$ admits cut-elimination.

Completeness for the asymmetric system. In this part, the translation of a hypersequent $G$ to a modal formula $\phi_{G}$ and the translation of a modal formula to a modal structure ()$^{s}$ are extensively used. Note that for two hypersequents $G$ and $H,\left(\phi_{G \mid H}\right)^{s}=\left[\left(\phi_{G}\right)^{s},\left(\phi_{H}\right)^{s}\right]$. For simplicity, we omit all $\perp$ disjuncts that occur in $\phi_{G}$. More importantly, since we treat only formulae in negation normal form, we extend the translation $\psi_{S}$ (used in $\phi_{G}$ ) to additionally push negation to atoms (according to the De Morgan dualities). For the same reason, system GS5 is modified in order to accommodate formulae with diamonds: the modal rules $(\vdash \square)$ and $(M S)$ take now the forms:

$$
\begin{gathered}
\frac{G|\square \Gamma \vdash \diamond \Delta, \alpha| H}{G|\square \Gamma \vdash \diamond \Delta, \square \alpha| H}(\vdash \square) \\
\frac{G\left|\square \Gamma_{1}, \diamond \Gamma_{2}, \Gamma_{3} \vdash \square \Delta_{1}, \diamond \Delta_{2}, \Delta_{3}\right| H}{G\left|\square \Gamma_{1}, \diamond \Gamma_{2} \vdash \square \Delta_{1}, \diamond \Delta_{2}\right| \Gamma_{3} \vdash \Delta_{3} \mid H}(M S)
\end{gathered}
$$

and the modal rules $(\diamond \vdash)$ and $(\vdash \diamond)$ are added to the system:

$$
\frac{G|\alpha, \square \Gamma \vdash \diamond \Delta| H}{G|\diamond \alpha, \square \Gamma \vdash \diamond \Delta| H}(\diamond \vdash) \quad \frac{G|\Gamma \vdash \Delta, \alpha| H}{G|\Gamma \vdash \Delta, \diamond \alpha| H}(\vdash \diamond)
$$

Furthermore, the rules for implication are dropped. We call the system obtained as described above by additionally removing the cut rule, system $\mathbf{G S 5}^{-}$. We will now show that every theorem of this system is also a theorem of system KSg-S5. We start with a lemma: 
Lemma 1 For a hypersequent $G$ and a structure $R$, the following rule is derivable in $\boldsymbol{K} \boldsymbol{S g}$-S5: $\quad g \downarrow \frac{S\left\{\square\left[R,\left(\phi_{G}\right)^{s}\right]\right\}}{S\left[\square R,\left(\phi_{G}\right)^{s}\right]}$

Proof By induction on the length of a hypersequent $G$.

Base step. $G$ is empty. Then $\left(\phi_{\emptyset}\right)^{s}=\mathrm{ff}$ and $S\{\square[R, \mathrm{ff}]\}=S\{\square R\}=$ $S[\square R, \mathbf{f f}]$.

Induction step. Let $G=G_{1}|\Gamma \vdash \Delta| H_{1}$. Then $\left(\phi_{G}\right)^{s}=\left[\square\left(\psi_{\Gamma \vdash \Delta}\right)^{s},\left(\phi_{G_{1} \mid H_{1}}\right)^{s}\right]$. By induction hypothesis we have $g \downarrow \frac{S\left\{\square\left[R,\left(\phi_{G_{1} \mid H_{1}}\right)^{s}\right]\right\}}{S\left[\square R,\left(\phi_{G_{1} \mid H_{1}}\right)^{s}\right]}$. Take the derivation

$$
\begin{gathered}
k \downarrow \frac{S\left\{\square\left[R,\left(\phi_{G_{1} \mid H_{1}}\right)^{s}, \square\left(\psi_{\Gamma \vdash \Delta}\right)^{s}\right]\right\}}{S\left[\square\left[R,\left(\phi_{G_{1} \mid H_{1}}\right)^{s}\right], \diamond \square\left(\psi_{\Gamma \vdash \Delta}\right)^{s}\right]} \\
5 \downarrow \frac{S\left[\square\left[R,\left(\phi_{G_{1} \mid H_{1}}\right)^{s}\right], \square\left(\psi_{\Gamma \vdash \Delta}\right)^{s}\right]}{g} \\
\quad \downarrow \frac{S\left[\square R,\left(\phi_{G_{1} \mid H_{1}}\right)^{s}, \square\left(\psi_{\Gamma \vdash \Delta}\right)^{s}\right]}{}
\end{gathered}
$$

Proposition $2 \vdash_{G S 5^{-}} G$ implies $\vdash_{K S g-S 5}\left(\phi_{G}\right)^{s}$.

Proof By induction on the length of a proof $\Pi$ of $G$, we show that the conclusion of every rule application in $\Pi$ is provable in $\mathbf{K S g}-\mathbf{S 5}$.

Base step. $G$ is an axiom. Then, $\phi_{\alpha \vdash \alpha}=\square(\neg \alpha \vee \alpha)$ and so $\left(\phi_{G}\right)^{s}=\square\left[\overline{\alpha^{s}}, \alpha^{s}\right]$. Then, $i \downarrow \frac{=\frac{\mathbb{t}}{\square t \mathbf{t t}}}{\square\left[\alpha^{s}, \alpha^{s}\right]}$ is a proof in KSg-S5.

Induction step. Here Lemma 1 is freely applied. Also, we use $\square \Gamma^{s}, \diamond \Gamma^{s}$ to abbreviate $\left[\square \Gamma_{1}^{s}, \ldots, \square \Gamma_{n}^{s}\right]$ and $\left[\diamond \Gamma_{1}^{s}, \ldots, \diamond \Gamma_{n}^{s}\right]$, respectively, for $\Gamma_{1}, \ldots, \Gamma_{n}$ the formulae in $\Gamma$.

1. Weakening. For the internal versions: by induction hypothesis there is a proof $\Pi^{\prime}$ of $\left[\square\left(\psi_{\Gamma \vdash \Delta}\right)^{s},\left(\phi_{G \mid H}\right)^{s}\right]$. Then, two different applications of $w \downarrow$ to it give $\left[\square\left[\bar{\alpha}^{s},\left(\psi_{\Gamma \vdash \Delta}\right)^{s}\right],\left(\phi_{G \mid H}\right)^{s}\right]$ and $\left[\square\left[\alpha^{s},\left(\psi_{\Gamma \vdash \Delta}\right)^{s}\right],\left(\phi_{G \mid H}\right)^{s}\right]$, as needed for left and right weakening, respectively.

For external weakening: by induction hypothesis there is a proof $\Pi^{\prime}$ of $\left(\phi_{G \mid H}\right)^{s}$. Then an application of $w \downarrow$ yields $\left[\square\left(\psi_{\Gamma \vdash \Delta}\right)^{s},\left(\phi_{G \mid H}\right)^{s}\right]$. 
2. Conjunction. For the left introduction rule: by induction hypothesis there is a proof of $\left[\square\left[\alpha^{s},\left(\psi_{\Gamma \vdash \Delta}\right)^{s}\right],\left(\phi_{G \mid H}\right)^{s}\right]$. Applying $w \downarrow$ yields a proof of $\left[\square\left[\overline{\alpha^{s}}, \overline{\beta^{s}},\left(\psi_{\Gamma \vdash \Delta}\right)^{s}\right],\left(\phi_{G \mid H}\right)^{s}\right]$, as it is needed. For the right rule: by induction hypothesis there are proofs $\Pi_{1}$ and $\Pi_{2}$ of $\left[\square\left[\alpha^{s},\left(\psi_{\Gamma \vdash \Delta}\right)^{s}\right],\left(\phi_{G \mid H}\right)^{s}\right]$ and $\left[\square\left[\beta^{s},\left(\psi_{\Gamma \vdash \Delta}\right)^{s}\right],\left(\phi_{G \mid H}\right)^{s}\right]$, respectively.

Then, for $S_{1}\{-\}=\square-$ and $S_{2}\{-\}=\square\left(\left[\square\left[\alpha^{s},\left(\psi_{\Gamma \vdash \Delta}\right)^{s}\right],\left(\phi_{G \mid H}\right)^{s}\right],-\right)$ build the proof

$$
\begin{gathered}
=\frac{\mathbb{t}}{\square t t} \\
\|\|_{S_{1}\left\{\Pi_{1}\right\} ; S_{2}\left\{\Pi_{2}\right\}} \\
s \frac{\square\left(\left[\square\left[\alpha^{s},\left(\psi_{\Gamma \vdash \Delta}\right)^{s}\right],\left(\phi_{G \mid H}\right)^{s}\right],\left[\square\left[\beta^{s},\left(\psi_{\Gamma \vdash \Delta}\right)^{s}\right],\left(\phi_{G \mid H}\right)^{s}\right]\right)}{\square\left[\left(\square\left[\alpha^{s},\left(\psi_{\Gamma \vdash \Delta}\right)^{s}\right],\left[\square\left[\beta^{s},\left(\psi_{\Gamma \vdash \Delta}\right)^{s}\right],\left(\phi_{G \mid H}\right)^{s}\right]\right),\left(\phi_{G \mid H}\right)^{s}\right]} \\
c \downarrow \frac{\square\left[\left(\square\left[\alpha^{s},\left(\psi_{\Gamma \vdash \Delta}\right)^{s}\right], \square\left[\beta^{s},\left(\psi_{\Gamma \vdash \Delta}\right)^{s}\right]\right),\left(\phi_{G \mid H}\right)^{s},\left(\phi_{G \mid H}\right)^{s}\right]}{\square\left[\left(\square\left[\alpha^{s},\left(\psi_{\Gamma \vdash \Delta}\right)^{s}\right], \square\left[\beta^{s},\left(\psi_{\Gamma \vdash \Delta}\right)^{s}\right]\right),\left(\phi_{G \mid H}\right)^{s}\right]} \\
t \downarrow \frac{\square\left[\left(\left[\alpha^{s},\left(\psi_{\Gamma \vdash \Delta}\right)^{s}\right], \square\left[\beta^{s},\left(\psi_{\Gamma \vdash \Delta}\right)^{s}\right]\right),\left(\phi_{G \mid H}\right)^{s}\right]}{\square\left[\left(\left[\alpha^{s},\left(\psi_{\Gamma \vdash \Delta}\right)^{s}\right],\left[\beta^{s},\left(\psi_{\Gamma \vdash \Delta}\right)^{s}\right]\right),\left(\phi_{G \mid H}\right)^{s}\right]} \\
s \frac{\square\left[\left(\left[\alpha^{s},\left(\psi_{\Gamma \vdash \Delta}\right)^{s}\right], \beta^{s}\right),\left(\psi_{\Gamma \vdash \Delta}\right)^{s},\left(\phi_{G \mid H}\right)^{s}\right]}{\square\left[\left(\alpha^{s}, \beta^{s}\right),\left(\psi_{\Gamma \vdash \Delta}\right)^{s},\left(\psi_{\Gamma \vdash \Delta}\right)^{s},\left(\phi_{G \mid H}\right)^{s}\right]} \\
c \downarrow \frac{\square\left[\left(\alpha^{s}, \beta^{s}\right),\left(\psi_{\Gamma \vdash \Delta}\right)^{s},\left(\phi_{G \mid H}\right)^{s}\right]}{\left[\square\left[\left(\alpha^{s}, \beta^{s}\right),\left(\psi_{\Gamma \vdash \Delta}\right)^{s}\right],\left(\phi_{G \mid H}\right)^{s}\right]}
\end{gathered}
$$

3. Modalized Splitting. By induction hypothesis there is a proof $\Pi^{\prime}$ of $\left[\square\left[\diamond \overline{\Gamma_{1}^{s}}, \square \overline{\Gamma_{2}^{s}}, \square \Delta_{1}^{s}, \diamond \Delta_{2}^{s},\left(\psi_{\Gamma_{3} \vdash \Delta_{3}}\right)^{s}\right],\left(\phi_{G \mid H}\right)^{s}\right]$. Then, for $S\{-\}=\square-$ build the proof

$$
\begin{aligned}
& =\frac{\mathrm{tt}}{\square \mathrm{tt}} \\
& \| S\left\{\Pi^{\prime}\right\} \\
& \begin{array}{l}
k^{*} \downarrow \frac{\square\left[\square\left[\diamond \overline{\Gamma_{1}^{s}}, \square \overline{\Gamma_{2}^{s}}, \square \Delta_{1}^{s}, \diamond \Delta_{2}^{s},\left(\psi_{\Gamma_{3} \vdash \Delta_{3}}\right)^{s}\right],\left(\phi_{G \mid H}\right)^{s}\right]}{\square\left[\diamond \diamond \overline{\Gamma_{1}^{s}}, \diamond \square \overline{\Gamma_{2}^{s}}, \diamond \square \Delta_{1}^{s}, \diamond \diamond \Delta_{2}^{s}, \square\left(\psi_{\Gamma_{3} \vdash \Delta_{3}}\right)^{s},\left(\phi_{G \mid H}\right)^{s}\right]} \\
4^{*} \downarrow \frac{\square\left[\nabla \overline{\Gamma_{1}^{s}}\right.}{\left.\diamond \square \overline{\Gamma_{2}^{s}}, \diamond \square \Delta_{1}^{s}, \diamond \Delta_{2}^{s}, \square\left(\psi_{\Gamma_{3} \vdash \Delta_{3}}\right)^{s},\left(\phi_{G \mid H}\right)^{s}\right]}
\end{array} \\
& 5^{*} \downarrow \frac{\square\left[\diamond \overline{\Gamma_{1}^{s}}, \diamond \square \overline{\Gamma_{2}^{s}}, \diamond \square \Delta_{1}^{s}, \diamond \Delta_{2}^{s}, \square\left(\psi_{\Gamma_{3} \vdash \Delta_{3}}\right)^{s},\left(\phi_{G \mid H}\right)^{s}\right]}{2} \\
& \square\left[\diamond \overline{\Gamma_{1}^{s}}, \square \overline{\Gamma_{2}^{s}}, \square \Delta_{1}^{s}, \diamond \Delta_{2}^{s}, \square\left(\psi_{\Gamma_{3} \vdash \Delta_{3}}\right)^{s},\left(\phi_{G \mid H}\right)^{s}\right] \\
& g \downarrow \overline{\left[\square\left[\diamond \overline{\Gamma_{1}^{s}}, \square \overline{\Gamma_{2}^{s}}, \square \Delta_{1}^{s}, \diamond \Delta_{2}^{s}, \square\left(\psi_{\Gamma_{3} \vdash \Delta_{3}}\right)^{s}\right],\left(\phi_{G \mid H}\right)^{s}\right]}
\end{aligned}
$$

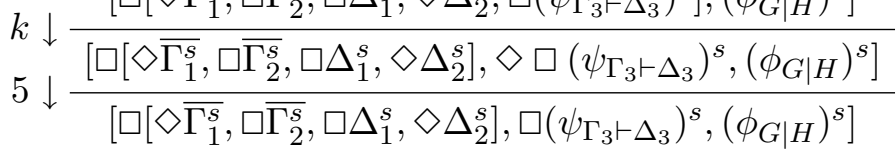


Note: The first three rules $k^{*} \downarrow, 4^{*} \downarrow$ and $5^{*} \downarrow$ abbreviate as many applications of $k \downarrow, 4 \downarrow$ and $5 \downarrow$ as there are formulae in $\left\{\Gamma_{1}, \Gamma_{2}, \Delta_{1}, \Delta_{2}\right\}$, $\left\{\Gamma_{1}, \Delta_{2}\right\}$ and $\left\{\Gamma_{2}, \Delta_{1}\right\}$, respectively.

4. Rules for $\square$. By induction hypothesis there are proofs $\Pi_{1}$ and $\Pi_{2}$ of $\left[\square\left[\alpha^{s},\left(\psi_{\Gamma \vdash \Delta}\right)^{s}\right],\left(\phi_{G \mid H}\right)^{s}\right]$ and $\left[\square\left[\diamond \overline{\Gamma^{s}}, \diamond \Delta^{s}, \alpha^{s}\right],\left(\phi_{G \mid H}\right)^{s}\right]$, respectively. Then, for $S\{-\}=\square-$ build the proof

$$
\begin{gathered}
=\frac{\mathbb{t t}}{\square t t} \\
\| S\left\{\Pi_{1}\right\}
\end{gathered}
$$

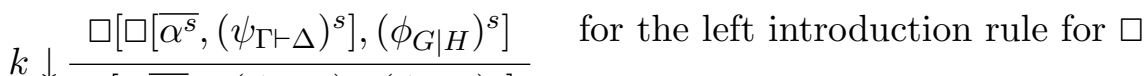

$$
\begin{aligned}
t & \downarrow \frac{\square\left[\diamond \overline{\alpha^{s}}, \square\left(\psi_{\Gamma \vdash \Delta}\right)^{s},\left(\phi_{G \mid H}\right)^{s}\right]}{\square\left[\diamond \overline{\alpha^{s}},\left(\psi_{\Gamma \vdash \Delta}\right)^{s},\left(\phi_{G \mid H}\right)^{s}\right]} \\
g & \downarrow \frac{\psi^{s},}{\left[\square\left[\diamond \overline{\alpha^{s}},\left(\psi_{\Gamma \vdash \Delta}\right)^{s}\right],\left(\phi_{G \mid H}\right)^{s}\right]}
\end{aligned}
$$

$$
\begin{aligned}
& =\frac{\mathrm{tt}}{\square \mathrm{tt}} \\
& \| S\left\{\Pi_{2}\right\}
\end{aligned}
$$

and the proof

$$
\begin{aligned}
k^{*} \downarrow & \frac{\square\left[\square\left[\diamond \overline{\Gamma^{s}}, \diamond \Delta^{s}, \alpha^{s}\right],\left(\phi_{G \mid H}\right)^{s}\right]}{\square\left[\diamond \diamond \overline{\Gamma^{s}}, \diamond \diamond \Delta^{s} \square \alpha^{s},\left(\phi_{G \mid H}\right)^{s}\right]} \\
4^{*} \downarrow & \text { for the right } \\
\quad g & \downarrow \frac{\square\left[\diamond \overline{\Gamma^{s}}, \diamond \Delta^{s}, \square \alpha^{s},\left(\phi_{G \mid H}\right)^{s}\right]}{\left[\square\left[\diamond \overline{\Gamma^{s}}, \diamond \Delta^{s}, \square \alpha^{s}\right],\left(\phi_{G \mid H}\right)^{s}\right]}
\end{aligned}
$$

introduction rules for $\square$. In the latter, the rules $k^{*} \downarrow$ and $4^{*} \downarrow$ abbreviate as many applications of $k \downarrow$ and $4 \downarrow$ as there are formulae in $\{\Gamma, \Delta\}$.

5. The cases of contraction, disjunction and the rules for $\diamond$ are similar to those of weakening, conjunction and the rules for $\square$, respectively. In the cases of negation, the results coincide with the induction hypothesis.

Theorem $3 \vdash_{S 5} \alpha$ implies $\vdash_{K S g-S 5} \alpha^{s}$.

Proof Since GS5 is complete for $S 5$, we have $\vdash_{G S 5} \vdash \alpha$ and so, $\vdash_{G S 5^{-}} \vdash \alpha$, which implies $\vdash_{K S g-S 5} \square\left(\psi_{\vdash \alpha}\right)^{s}$ (Proposition 2). Since $\alpha$ is a single formula, we have $\vdash_{K S g-S 5} \square \alpha^{s}$. Applying $t \downarrow$ yields $\vdash_{K S g-S 5} \alpha^{s}$, as needed.

Corollary 1 System $\mathbf{K S g}-\boldsymbol{S} 5$ is complete for $S 5$. 
Corollary $\mathbf{2}$ The up-fragment of system $\boldsymbol{S K S g - S \boldsymbol { 5 }}$ is admissible.

\section{Conclusions and Future Directions}

We have presented a system for $S 5$ in the calculus of structures, a formalism that makes explicit use of deep inference. The system comprises modal rules that are in exact correspondence to each of the axioms in $\{K, T, 4,5\}$. Cutadmissibility has been obtained via embedding of cut-free proofs from the hypersequent system for $S 5$ (Avron [1]). The simple design of our system and the clear correspondence of its modal rules to their axioms diversify it from other systems developed so far for $S 5$. Furthermore, the system can be restricted to its local variant, in which rule applications affect only structures of bounded length. This local system (Stouppa [28]) is obtained in a similar way to the local system for predicate logic, system $S K S q$ (Brünnler [4]).

Our system comprises a rule for the 4 axiom, an axiom which is derivable in $S 5$. We believe that its presence is only justified by the method used in obtaining cut-admissibility, and that it does not affect the set of theorems of the system. Thus, as a matter of future work we shall investigate its admissibility in the cut-free system for $S 5$ which does not include the 4-rule. Evidently, the rule is derivable in the symmetric variant of the system in consideration. The above conjecture will immediately follow from a syntactic proof of cut-elimination. Such a result would be also crucial for the establishment of the calculus of structures as a suitable formalism for the proof analysis of modal logics. A further direction will be then the formulation of a cut-free system for logic $B$. A system for this logic has been already formulated in the calculus of structures (Hein [9]), however its cut-admissibility is only conjectured.

Another important research direction is the development of efficient proof search procedures for the modal systems in the calculus of structures. System implementations for this formalism have been already developed (Kahramanoğulları $[13,14,15]$ ) and concern systems for propositional logic and variants of linear logic. We expect that some of the techniques applied in those systems will also apply in the modal systems. Apart from the system for $S 5$, cut-free modal systems developed in this formalism include systems for the logics $K, M$ and $S 4$ and have been presented in Stewart and Stouppa [27]. An up-to-date state of developments in the calculus of structures can be found under

http://alessio.guglielmi.name/res/cos/index.html . 


\section{References}

[1] A. Avron. The Method of Hypersequents in the Proof Theory of Propositional Non-classical Logics. In W. Hodges, M. Hyland, C. Steinhorn and J. Truss, editors, Logic: From Foundations to Applications, pages 1-32. Oxford University Press, Oxford, 1996.

[2] T. Braüner. A cut-free Gentzen formulation of the modal logic S5. In the Logic Journal of the Interest Group in Pure and Applied Logics, volume 8(5), pages 629-643, 2000.

[3] T. Braüner. Functional completeness for a natural deduction formulation of hybridized S5. In P. Balbiani, N.-Y. Suzuki, F. Wolter and M. Zakharyaschev, editors, Advances in Modal Logic, volume 4, pages 31-49. King's College Publications, 2003.

[4] K. Brünnler. Deep Inference and Symmetry in Classical Proofs. PhD thesis, Technische Universität Dresden, 2003.

[5] M. Fitting. A simple propositional S5 tableau system. In the Annals of Pure and Applied Logic, volume 96, pages 107-115, 1999.

[6] G. Gentzen. Investigations into logical deduction. In M. E. Szabo, editor, The Collected Papers of Gerhard Gentzen, pages 68-131. NorthHolland, Amsterdam, 1969.

[7] A. Guglielmi. A System of Interaction and Structure. Technical report WV-02-10, Technische Universität Dresden, 2002. To appear in ACM Transactions on Computational Logic. Preprint available at http://iccl.tu-dresden.de/ guglielm/p/SystIntStr.pdf .

[8] A. Guglielmi and L. Straßburger. Non-commutativity and MELL in the Calculus of Structures. In L. Fribourg, editor, CSL 2001, LNCS 2142, pages 54-68, Springer-Verlag, 2001.

[9] R. Hein. Geometric Theories and Modal Logic in the Calculus of Structures. Master Thesis, Technische Universität Dresden, 2005.

[10] G. Hughes and M. Cresswell. A New Introduction to Modal Logic. Routledge, 1996.

[11] A. Indrzejczak. Cut-free Double Sequent Calculus for S5. In the Logic Journal of the Interest Group in Pure and Applied Logics, volume 6(3), pages 505-516, 1998. 
[12] A. Indrzejczak. Generalised Sequent Calculus for Propositional Modal Logics. In Logica Trianguli, volume 1, pages 15-31, 1997.

[13] O. Kahramanoğulları. Implementing System BV of the Calculus of Structures in Maude. In L. Alonso i Alemany and P. Égré, editors, Proceedings of the ESSLLI-2004 Student Session, pages 117-127, 2004.

[14] O. Kahramanoğulları. Reducing Nondeterminism in the Calculus of Structures. Technical Report WV-06-01, Technische Universität Dresden, 2006.

[15] O. Kahramanoğulları. System BV without the Equalities for Unit. In C. Aykanat, T. Dayar, I. Korpeoglu, editors, ISCIS'04, LNCS 3280, pages 986-995, Springer-Verlag, 2004.

[16] S. Kanger. Provability in Logic. Almqvist \& Wiksell, Stockholm, 1957.

[17] E. Lemmon and D. Scott. An Introduction to Modal Logic. Oxford:Blackwell, 1977.

[18] G. Mints. A Short Introduction to Modal Logic. CSLI Lecture Notes 30, CSLI Publications, Stanford, 1992.

[19] G. Mints. Lewis' systems and system T. In Selected Papers in Proof Theory, pages 221-294. Bibliopolis, North-Holland, 1992.

[20] S. Negri. Proof analysis in modal logic. To appear in the Journal of Philosophical Logic.

[21] M. Ohnishi and K. Matsumoto. Gentzen method in modal calculi, parts I and II. In Osaka Mathematical Journal, volume 9, pages 113130, 1957, and volume 11, pages 115-120, 1959.

[22] E. Orlowska. Relational Proof Systems for Modal Logics. In H. Wansing, editor, Proof Theory of Modal Logic, pages 55-77. Kluwer Academic Publishers, Dordrecht, 1996.

[23] G. Pottinger. Uniform, Cut-free formulations of T, S4 and S5, abstract. In the Journal of Symbolic Logic, volume 48, page 900, 1983.

[24] M. Sato. A Study of Kripke-type Models for Some Modal Logics by Gentzen's Sequential Method. Publications of the Research Institute for Mathematical Sciences, Kyoto University, volume 13, pages 381468, 1977. 
[25] G. F. Shvarts. Gentzen style systems for K45 and K45D. In A. R. Meyer and M. A. Taitslin, editors, Logic at Botik'89, LNCS 363, pages 245-256, Springer, Berlin, 1989.

[26] A. Simpson. The Proof Theory and Semantics of Intuitionistic Modal Logic. PhD thesis, University of Edinburgh, 1994.

[27] C. Stewart and P. Stouppa. A systematic proof theory for several modal logics. In R. Schmidt, I. Pratt-Hartmann, M. Reynolds and H. Wansing, editors, Advances in Modal Logic, volume 5, pages 309333, King's College Publications, 2005.

[28] P. Stouppa. The Design of Modal Proof Theories: the case of S5. Master Thesis, Technische Universität Dresden, 2004.

[29] L. Straßburger. A Local System for Linear Logic. In M. Baaz and A. Voronkov, editors, LPAR 2002, LNAI 2514, pages 388-402, Springer-Verlag, 2002.

[30] H. Wansing. Displaying Modal Logic Kluwer Academic Publishers, 1998.

[31] H. Wansing. Translation of Hypersequents into Display Sequents. In the Logic Journal of the Interest Group in Pure and Applied Logics, volume 6(5), pages 719-733, 1998. 